\title{
Functional Heterogeneity in Human Olfactory Cortex: An Event- Related Functional Magnetic Resonance Imaging Study
}

\author{
Jay A. Gottfried, Ralf Deichmann, Joel S. Winston, and Raymond J. Dolan \\ Wellcome Department of Imaging Neuroscience, Institute of Neurology, London, WC1N 3BG, United Kingdom
}

\begin{abstract}
Studies of patients with focal brain injury indicate that smell perception involves caudal orbitofrontal and medial temporal cortices, but a more precise functional organization has not been characterized. In addition, although it is believed that odors are potent triggers of emotion, support for an anatomical association is scant. We sought to define the neural substrates of human olfactory information processing and determine how these are modulated by affective properties of odors. We used event-related functional magnetic resonance imaging (fMRI) in an olfactory version of a classical conditioning paradigm, whereby neutral faces were paired with pleasant, neutral, or unpleasant odors, under 50\% reinforcement. By comparing paired (odor/face) and unpaired (face only) conditions, odorevoked neural activations could be isolated specifically. In primary olfactory (piriform) cortex, spatially and temporally disso-
\end{abstract}

How odors access central brain structures has been well characterized in animal models (Carmichael et al., 1994; Haberly, 1998). Odor-evoked responses are conducted from olfactory receptor neurons at the nasal mucosa toward the olfactory bulb. Secondorder projections transmit via the lateral olfactory tract and terminate in adjacent structures collectively labeled "primary olfactory cortex." Piriform cortex is the major recipient of bulbar afferents, but additional targets comprise olfactory tubercle, anterior olfactory nucleus, periamygdaloid cortex, and entorhinal area. Higher-order projections converge on orbitofrontal cortex (OFC), agranular insula, mediodorsal thalamus, and hypothalamus (Haberly, 1998). Although each of these anatomical zones participates differentially in olfactory processing, it is apparent that the piriform cortex may be functionally heterogeneous, particularly along its rostral-caudal extent (Litaudon et al., 1997; Haberly, 1998).

The neurobiology of human olfaction has received less attention compared with other sensory modalities. It is generally agreed that the human sense of smell involves posterior orbitofrontal and anteromedial temporal lobes (Eslinger et al., 1982). Studies of patients with brain damage to these regions reveal defects in odor identification, discrimination, and memory (Potter and Butters, 1980; Eskenazi et al., 1983; Zatorre and Jones-

Received July 16, 2002; revised Sept. 23, 2002; accepted Sept. 27, 2002.

This research was supported by a Physician Postdoctoral Fellowship from the Howard Hughes Medical Institute (J.A.G.) and by a Wellcome Trust Programme Grant (R.J.D.). We are grateful to P. Aston, E. Featherstone, and O. Josephs for design and construction of the olfactometer, and to D. Corfield and J. O'Doherty for helpful discussions. We also thank the radiology staff of the Wellcome Department of Imaging Neuroscience.

Correspondence should be addressed to Dr. Jay A. Gottfried, Wellcome Department of Imaging Neuroscience, Functional Imaging Laboratory, 12 Queen Square, London WC1N 3BG, UK. E-mail: j.gottfried@fil.ion.ucl.ac.uk.

Copyright (C) 2002 Society for Neuroscience $0270-6474 / 02 / 2210819-10 \$ 15.00 / 0$ ciable responses were identified along a rostrocaudal axis. A nonhabituating response in posterior piriform cortex was tuned to all odors, whereas activity in anterior piriform cortex reflected sensitivity to odor affect. Bilateral amygdala activation was elicited by all odors, regardless of valence. In posterior orbitofrontal cortex, neural responses evoked by pleasant and unpleasant odors were segregated within medial and lateral segments, respectively. The results indicate functional heterogeneity in areas critical to human olfaction. They also show that brain regions mediating emotional processing are differentially activated by odor valence, providing evidence for a close anatomical coupling between olfactory and emotional processes.

Key words: olfaction; odor; emotion; neuroimaging; fMRI; piriform cortex; olfactory cortex; amygdala; orbitofrontal cortex
Gotman, 1991). However, the large size and spatial extent of such lesions preclude careful structural delineation, especially given the close proximity of so many critical regions. Thus, despite the abundance of clinical data, a more precise functional organization of human olfaction has not been elucidated.

Neuroimaging studies have begun to identify important olfactory structures, but one notable feature is the inconsistent activation of piriform cortex (Zald and Pardo, 2000). This may reflect two factors. First, conventional functional magnetic resonance imaging (fMRI) sequences are associated with signal loss (susceptibility artifact) at air-tissue interfaces, reducing image quality in ventral temporal areas, specifically in piriform cortex (Ojemann et al., 1997). Second, olfactory habituation occurs with prolonged odor exposure in rodent piriform cortex (Wilson, 1998), and analogous phenomena have been confirmed with fMRI in humans (Sobel et al., 2000; Poellinger et al., 2001). Because many previous olfactory neuroimaging studies used blocked designs, with constant odor presentation over 30-60 sec, habituation has been an unavoidable confound.

To overcome these difficulties, we combined event-related techniques with a novel fMRI acquisition sequence that reduced signal dropout. Our principal objective was to define regions responsive to olfactory stimulation and odor valence. Olfactory perception is thought to be dominated by hedonic (pleasurable) properties (Schiffman, 1974), and it is commonly held that odors are potent emotional cues. Thus, we hypothesized that if the olfactory system is sensitive to hedonics, then manipulations of odor valence should highlight differences within olfactory regions and elicit activations in structures implicated in emotional processing, such as amygdala and OFC. Here we describe the functional neuroanatomy of human olfaction, with specific reference to dissociations between unpleasant and pleasant odors. A com- 
panion paper in this issue (Gottfried et al., 2002) focuses on the neural components of olfactory learning.

\section{MATERIALS AND METHODS}

Subjects. Informed consent was obtained from 17 healthy, right-handed subjects (10 women; age range, $18-31$ years; mean age, 23 years), who were recruited by advertisement. No subject had a history of neurological or psychiatric illness, respiratory or ear-nose-throat problems, or known defects of smelling. The study was devised in accordance with the rules and regulations of the joint National Hospital for Neurology and Neurosurgery and Institute of Neurology Ethics Committee. The data from two subjects (one woman) were rejected because of technical failures during the scanning procedure.

Experimental paradigm and stimuli. Subjects participated in an olfactory version of classical conditioning in which a neutral item [the conditioned stimulus $(\mathrm{CS}+)$ ] acquires behavioral significance by predicting the occurrence of an emotionally salient item [the unconditioned stimulus (UCS)] after repeated pairings. In this instance, a series of neutral faces represented the $\mathrm{CS}+$, and three different odors varying in pleasantness were used as the UCS. Although the paradigm enabled us to investigate olfactory-visual associative learning (Gottfried et al., 2002), it also permitted an independent analysis of olfactory sensory processing, to provide an account of the functional anatomy of human olfaction unhindered by previous methodological limitations.

Four neutral, grayscale faces (two male, two female) taken from the Ekman series of facial affect (Ekman and Friesen, 1976) comprised the one nonconditioned ( $\mathrm{CS}-$ ) and 3 conditioned (CS+) stimuli. All facial hair was removed from the pictures to increase task difficulty (see below). Images were 150 pixels wide $\times 205$ pixels high and subtended visual angles of $\sim 8 \times 10^{\circ}$ when back-projected onto the display screen of a head box. Subjects viewed the faces in the center of a gray background.

The odor stimuli were selected as follows. In a pilot study involving 20 different odors (Sigma-Aldrich Company Ltd., Dorset, UK), visual analog ratings of odor valence were collected from an independent group of 12 subjects outside the scanner. Three odors were identified that varied widely in perceived pleasantness, could be easily distinguished from each other, and showed good intersubject agreement. These were as follows: vanillin (VAN; $8 \% \mathrm{w} / \mathrm{v}$ in propylene glycol), the pleasant odor; phenethyl alcohol (PEA; $0.1 \% \mathrm{v} / \mathrm{v}$ in propylene glycol), the neutrally valenced odor; and 4-methyl-pentanoic acid (4MP; 5\% v/v in mineral oil), the unpleasant (aversive) odor. At the concentrations used, these compounds have been shown to be relatively specific to olfactory nerve (cranial nerve I) stimulation, with minimal activation of trigeminal (cranial nerve V) pathways (Doty et al., 1978).

Odor delivery. Odors were delivered via a four-channel, computercontrolled olfactometer that was constructed after a design by Lorig et al. (1999), with the exception that air dilution channels were not included. The apparatus is suitable for the MRI environment because all metal components are outside the scanner room, and it generates discrete pulses of odor with a rapid on-off time on the order of $250-500 \mathrm{msec}$. In addition, the system is free of tactile, thermal, or auditory shifts that might otherwise interfere with task demands or mental set. Under baseline conditions, room air is normally conveyed to the subject through a nasal cannula nosepiece. At odor onset, a computer signal simultaneously toggles a control (air) valve off and an odor valve on, resulting in the administration of odorized air to the subject. At odor offset, the signal sequence is reversed, which reintroduces room air and guarantees rapid washout of residual odor within the system. Airflow was kept constant at $2.5 \mathrm{l} / \mathrm{min}$. Both the presentation of the visual stimuli and the triggering of the odor valves were accomplished using Cogent 2000 software (Wellcome Department of Imaging Neuroscience, London, UK), as implemented in Matlab 6.0 (The Mathworks Inc., Natick, MA).

Respiratory monitoring. Subjects were pretrained to make an appropriate sniff with instruction to maintain the same volume and rate for each trial. Because the act of sniffing induces activations within primary olfactory cortex (Sobel et al., 1998), the breathing patterns of each subject were monitored on-line during the experiment to ensure that sniffing was kept constant across all event types. A pair of breathing belts made of corrugated rubber tubing (Siemens, Erlangen, Germany) was affixed around the chest and abdomen. As the subject breathed, pressure changes within the rubber tubing (caused by chest and abdominal movements) could be detected by means of a piezo-resistive differential pressure sensor ( $0-1$ psi; Honeywell, Morristown, NJ) positioned outside the scanner room. This signal was subsequently sampled at $100 \mathrm{~Hz}$ and recorded digitally on a PC computer using Spike2 software (version 3.16,
Cambridge Electronic Design Ltd., Cambridge, UK). Subject-specific sniff waveforms were pooled across each condition and adjusted by subtracting the mean activity in the $400 \mathrm{msec}$ preceding sniff onset to account for baseline fluctuations. These were then normalized to the peak amplitude of the CS - condition to permit analysis at the group level. In addition, temporal trends in the data were investigated by performing linear regression (least squares) on subject-specific peak amplitudes independently for each condition. The estimated regression slopes were used to quantify the degree of change in sniff amplitude over time, which then could be averaged across subjects for statistical analysis.

Task. Subjects were not informed about face-odor contingencies but were simply asked to make a forced-choice pushbutton response regarding facial gender. At the start of the experiment, and between successive trials, a dull-red cross-hair appeared on a gray background. The onset of a given trial was heralded by the appearance of a face just above the cross-hair $(t=0)$. After $500 \mathrm{msec}(t=500)$, the cross-hair turned bright red as a prompt to sniff, and the olfactometer was triggered to deliver an odor or control air, depending on trial type (Fig. 1A). The sniff cue remained bright for $750 \mathrm{msec}(t=1250)$, after which time it returned to its dull red color (sniff stop). It has been shown that single sniffs of this duration are optimal for odor detection (Laing, 1986). Note that the face and odor overlap for $250 \mathrm{msec}$ and that the offset of the face $(t=750)$ always occurs before the offset of the sniff cue $(t=1250)$. Also note that subjects complete a sniff on each and every trial, regardless of odor presence. An intertrial interval of $7.5 \mathrm{sec}$ allowed the subject to take one to two regular breaths in between sniffs and was sufficient to clear residual odor from the olfactometer and the nasal passages.

During the experiment, $50 \%$ of all face $(\mathrm{CS}+)$ presentations were paired with their corresponding odor (UCS) under a partialreinforcement strategy. This allowed us to model event-related responses specific to the compound response (paired face/odor) separately from the unpaired CS + (face only) and resulted in seven unique event-types: (1) "appetitive" face, paired with pleasant odor $\left(\operatorname{appCS}+_{P}\right)$; $(2)$ appetitive face, unpaired (appCS $\left.+_{U}\right)$; (3) "neutral" face, paired with neutral odor $\left(\right.$ ntCS $\left.+_{\mathrm{P}}\right) ;(4)$ neutral face, unpaired $\left(\mathrm{ntCS}+_{\mathrm{U}}\right) ;(5)$ "aversive" face, paired with unpleasant odor $\left(\operatorname{avCS}+_{\mathrm{P}}\right) ;(6)$ aversive face, unpaired $\left(\operatorname{avCS}+_{U}\right)$; and (7) $\mathrm{CS}-($ face never paired with odor) (Fig. 1A). For a given subject, the same face was always paired with the same odor, but face-odor combinations were counterbalanced across subjects. There were 25 replications of each event type, except for the CS-, which was repeated 50 times, and the order of stimulus presentations was randomized. On the basis of an experimental length of $25 \mathrm{~min}$, a given odor was repeated on average every $50-60 \mathrm{sec}$, an interval found to mitigate olfactory habituation (Kobal and Hummel, 1991).

Image acquisition. Gradient echo $\mathrm{T} 2 *$-weighted echoplanar images (EPI) were acquired with blood-oxygen level-dependent (BOLD) contrast on a 2-Tesla Siemens Vision MRI scanner (Siemens, Erlangen, Germany), using a novel combination of image tilting and $z$-shimming to improve functional sensitivity in orbitofrontal and medial temporal regions (Deichmann and Turner, 2002). Briefly, multislice EPI datasets were acquired in an oblique orientation $30^{\circ}$ to the anterior commissureposterior commissure line (rostral $>$ caudal), with the following parameters: echo time, $35 \mathrm{msec}$; field-of-view, $192 \mathrm{~mm}$; in-plane resolution, 3.0 $\mathrm{mm}$. Because of the oblique slice orientation, signal losses caused by susceptibility gradients in phase-encoding direction could be avoided. Additionally, spin dephasing attributable to through plane susceptibility gradients was reduced by including a preparation pulse with a duration of $1 \mathrm{msec}$ and an amplitude of $-2 \mathrm{mT} / \mathrm{m}$ in slice selection direction directly before data acquisition, similar to $z$-shimming (Constable and Spencer, 1999). In contrast to standard $z$-shimming, however, the new technique does not require the combination of several images. Thus, the temporal resolution is not compromised.

Volumes consisted of 33 slices $(1.8 \mathrm{~mm}$ thickness, $1.2 \mathrm{~mm}$ gap) that covered $\sim 80 \%$ of the whole brain, apart from superior aspects of the parietal lobes (Fig. $1 B$ ), and they were collected continuously every 2.31 sec. The entire data set was then reconstructed into conventional threedimensional (untilted) space using trajectory-based reconstruction (Josephs et al., 2000) after a trajectory scan was calibrated at $30^{\circ}$ during a gel-phantom experiment. High-resolution T1-weighted anatomical images with $1 \mathrm{~mm}$ in-plane resolution were also acquired for each subject.

Subject debriefing. Post hoc ratings of odor valence were collected from each subject outside the scanner, using a visual analog scale (16 mm line) delimited with anchors signifying "extremely pleasant" and "extremely unpleasant." Ratings of odor intensity were acquired similarly, with endpoints representing "undetectable" and "extremely strong." 
A.
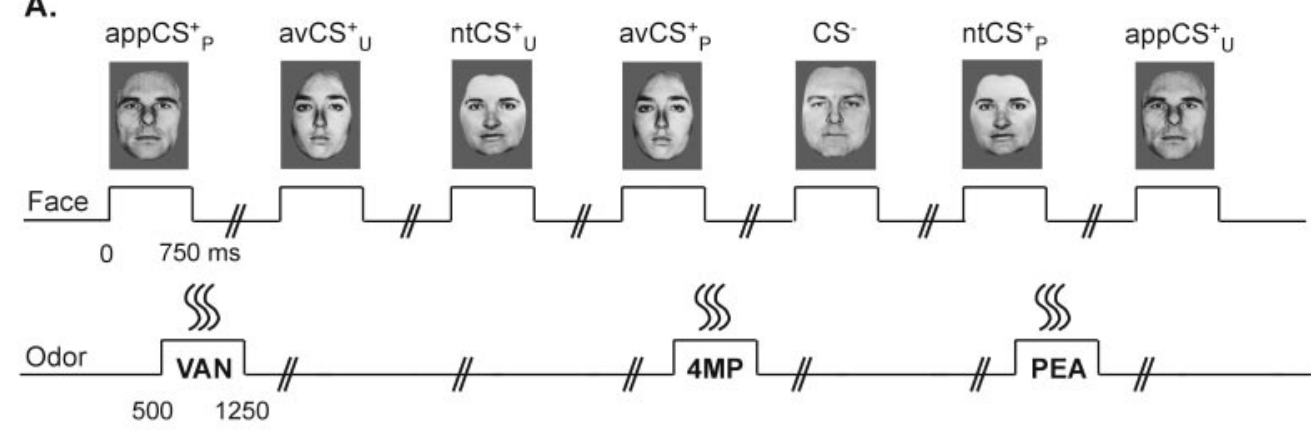

Figure 1. Task and imaging protocol. $A$, A series of neutral faces (top row) was paired with odors (middle row) that varied in pleasantness. Under $50 \%$ reinforcement, only one-half of all faces were paired with odor. A brightening of a cross-hair (bottom row, + ) was used to prompt the subject to sniff. The four faces and three odors comprised seven different event types and are depicted schematically. See Materials and Meth-

B.
Imaging acquisition plane

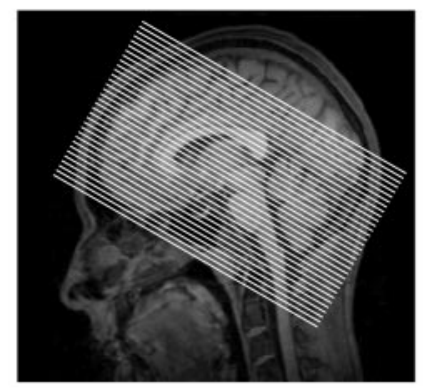

Tilted sequence

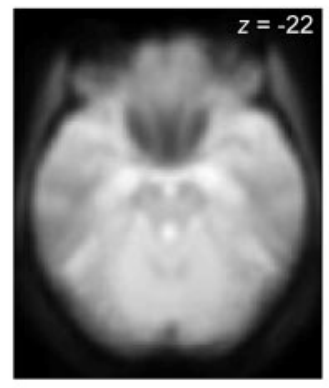

Standard sequence

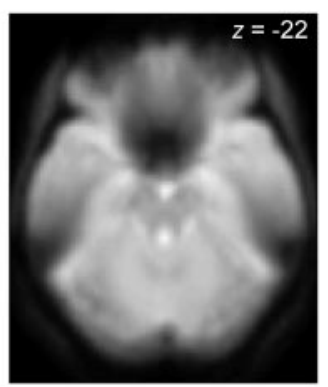
ods for abbreviations and further details. $B$, Functional images were acquired in an oblique orientation tilted at $30^{\circ}$. On the left, the plane of acquisition and the effective brain coverage are shown overlaid on a subject's sagittal T1-weighted scan. The resulting echoplanar image $(z=-22)$ averaged from 15 subjects is shown beside an image obtained using a standard (untilted) acquisition sequence (average of 11 subjects). Preservation of signal is apparent in the basal frontal and medial temporal lobes. Signal dropout is also diminished in lateral posterior temporal areas.
Image preprocessing. A total of 650 image volumes were collected from each subject. After discarding the first six volumes ("dummy scans") to allow for T1-equilibration effects, functional images were realigned to the first volume to correct for subject motion (Friston et al., 1995a) and then slice-time corrected to the middle slice of each volume. This generated a set of volume-specific movement parameters included as regressors of no interest in subsequent models of the data. The realigned images were then spatially normalized into a standard anatomical space, which permitted data analysis at the group level, and smoothed with an $8 \mathrm{~mm}$ (full-width half-maximum) Gaussian kernel, to account for residual intersubject differences. Each of the subject's structural T1 scans was transformed into EPI space by coregistration to the mean functional image and then spatially normalized using the parameters derived from EPI normalization.

Analysis of olfactory-evoked responses. Event-related fMRI data were analyzed using Statistical Parametric Mapping (SPM99, Wellcome Department of Imaging Neuroscience) in the context of a random-effects model. First, events were modeled by a set of delta (stick) functions that corresponded to the onset times of each face for each of the seven event types (i.e., $\operatorname{appCS}_{\mathrm{P}}, \operatorname{appCS}+_{\mathrm{U}}, \mathrm{ntCS}+_{\mathrm{P}}, \mathrm{ntCS}+_{\mathrm{U}}, \operatorname{avCS}+_{\mathrm{P}}$, avCS $+_{\mathrm{U}}$, and $\mathrm{CS}-$ ). Regressors of interest were then created by convolving the $\delta$ functions with a canonical hemodynamic response function (HRF) along with its temporal and spatial derivatives to accommodate shifts in response latency and dispersion (Friston et al., 1998). The influence of olfactory habituation was also incorporated into the model as condition $\times$ time interactions by multiplying each regressor of interest by a mean-corrected exponential function, using a time-constant (one-eighth session length, or $186 \mathrm{sec}$ ) similar to that demonstrated in previous olfactory neuroimaging studies (Sobel et al., 2000; Poellinger et al., 2001). Movement parameters were entered into the model as effects of no interest, as were low-frequency drifts in signal (cutoff $120 \mathrm{sec}$ ). Condition-specific parameter estimates (pertaining to the height of the HRF) were calculated independently for each brain voxel using the general linear model (Friston et al., 1995b). Contrasts of parameter estimates from the 15 subjects were subsequently entered into one-way $t$ tests (random-effects analysis), each of which constituted an SPM $\{\mathrm{T}\}$. Odor-evoked neural responses were specifically isolated by conducting comparisons between paired and unpaired CS + conditions. For example, the contrast of $\left(\right.$ appCS $\left.+_{\mathrm{P}}-\operatorname{appCS}+_{\mathrm{U}}\right)$ was performed to highlight activations specific to the pleasant odor. In other words, the unpaired CS + events served as a useful olfactory baseline or control by canceling out nonspecific effects of visual stimulation (face and cross-hair), sniffing, and motor (button press) activity.

Regions of activation were localized using the human brain atlas of Duvernoy (1999) and Mai et al. (1997). In areas where odor-evoked responses were predicted a priori (namely, primary and secondary olfactory projection zones, including amygdala, OFC, insula, hypothalamus, and mediodorsal thalamus), we report activations surviving a threshold of $p<$ 0.001 uncorrected, although in piriform and entorhinal cortex, a more liberal threshold of $p<0.005$ uncorrected was used (Ojemann et al., 1997). A correction for multiple comparisons was also performed across small volumes of interest (Worsley et al., 1996) by constructing bilateral anatomical masks of each region using MRIcro software (Rorden and Brett, 2000), with reference to a normalized T1-weighted structural scan, to limit the effective search space. In mediodorsal thalamus, a sphere of $10 \mathrm{~mm}$ radius was positioned at its center $(0,-20,+6)$. The statistical threshold used to report these activations was set at $p<0.05$ corrected. All reported voxels of interest conform to the Montreal Neurological Institute (MNI) template coordinate system.

A conjunction analysis examined brain regions commonly activated by all odors (Price and Friston, 1997). First, subject-specific parameter estimates were calculated for each of six effects of interest: the three paired CS+ conditions (appetitive, neutral, aversive) and their three respective unpaired CS + baselines. Second, these estimates from all 15 subjects were entered into a repeated-measures ANOVA using a correction for nonsphericity (Glaser et al., 2002). This procedure is based on the assumptions that within-subject variance is not identical across conditions (e.g., a given subject might adopt different cognitive strategies between paired and unpaired events) and that between-subject covariance is not independent over conditions (e.g., one subject might be a better "smeller" than another). By testing linear contrasts of each paired $\mathrm{CS}+$ relative to its corresponding unpaired $\mathrm{CS}+$, we were able to isolate odor-evoked neural responses independent of hedonic context. This process was also applied to the set of six regressors parametrically modulated by time.

Finally, an illustrative model of the data were constructed to depict the time course of activation within (piriform) voxels identified as significant in the primary model. By subdividing the 25 events of a given condition into 
six blocks of four (or five) temporally contiguous events, six regressors of interest were defined for each condition type. Subsequent analysis was performed identically to the primary model, except that condition $\times$ time interactions were not included. By testing linear contrasts of parameter estimates between paired and unpaired conditions for each of the six blocks, the response profile of a given voxel across time could be assessed. Because of the inherently low signal-to-noise ratio of individual eventrelated responses, data were displayed as means of parameter estimates for every two successive blocks (that is, mean of first and second block, mean of third and fourth block, mean of fifth and sixth block).

\section{RESULTS}

\section{Behavioral}

None of the subjects reported change in odor valence or intensity over the course of the experiment. One subject thought that VAN became slightly less pleasant over time, so her score represented the average of two ratings based on the start and end of scanning. Mean pleasantness ratings $(-8$ to +8$)$ for the three odors were as follows: VAN, $3.29 \pm 0.75$ ( \pm SEM); PEA, $0.82 \pm 0.86$; and 4MP, $-5.01 \pm 0.62$ (Fig. $2 A$ ). There was a significant difference among these ratings, as determined by a Friedman Test for related samples $\left(\chi^{2}=20.133 ; \mathrm{df}=2 ; p<0.001\right)$. Pairwise post hoc comparisons showed that VAN was perceived as significantly more pleasant than both PEA and 4MP and that 4MP was judged to be significantly less pleasant than PEA $(p<0.05$; one-tailed Wilcoxon signed ranks test). Mean ratings of odor intensity (0 to +16 ) revealed the following: VAN, $8.95+0.79$; PEA, $7.45+$ 0.77 ; and $4 \mathrm{MP}, 11.72+1.10$ (Fig. $2 B$ ). There was a significant trend toward differences between intensity ratings $\left(\chi^{2}=8.169\right.$; $\mathrm{df}=2 ; p=0.017$; Friedman Test), and in pairwise tests, 4MP was judged as significantly more intense than VAN and PEA $(p<$ 0.05 ; one-tailed Wilcoxon Test), but the intensity of VAN did not differ significantly from that of PEA $(p=0.3)$.

Analysis of sniff waveforms was pooled across all subjects for each condition type. The condition-specific responses of a single subject are shown (Fig. $2 C$ ), and the group mean amplitudes and latencies are charted (Figs. 2D,E). Although there was a slight amplitude decrement (17\%) in sniffing the unpleasant odor $\left(\right.$ avCS $\left.+_{\mathrm{P}}\right)$ relative to the $\mathrm{CS}-$ as well as a mild increment $(8 \%)$ in sniffing the pleasant odor $\left(\operatorname{appCS}{ }_{\mathrm{P}}\right)$, there was no significant difference in the peak sniff amplitude between any of these conditions $(F=2.149$; $\mathrm{df}=1.54 ; p=0.149$; one-way repeated-measures ANOVA; df adjusted by the Greenhouse-Geisser procedure). There was also no significant difference in sniff latency (measured from sniff-cue onset to time-to-peak) among the various conditions $(F=0.956 ; \mathrm{df}=1.91 ; p=0.394)$. Finally, no significant temporal trends in peak sniff amplitude were observed for any of the seven conditions, as determined by regression analysis. Specifically, there was no significant difference between the mean regression slopes among the different conditions $(F=1.102$; df $=1.95 ; p=0.345$; one-way repeated-measures ANOVA; df adjusted), nor did the mean regression slopes for any single condition differ significantly from zero in a series of one-sample $t$ tests (all $p>0.1$; two-tailed).

\section{Neuroimaging}

\section{Valence-independent odor activations}

Conjunction analysis permitted an evaluation of brain regions activated by all odors, independent of hedonic context. The combination of $\left(\operatorname{appCS}+_{\mathrm{P}}-\operatorname{appCS}+_{\mathrm{U}}\right)$ and $\left(\mathrm{ntCS}+_{\mathrm{P}}-\right.$ $\left.n t C S+_{U}\right)$ and $\left(\operatorname{avCS}+_{P}-\operatorname{avCS}+_{U}\right)$ showed significant activations in piriform cortex bilaterally along its posterior aspect (Fig. $3 A, D, i)$. On the left side, this response spanned both frontal and temporal piriform areas $(x, y, z$ coordinates: $-32,4,-20 ; p<0.05$ corrected for small volumes (SVC); and $-26,2,-26 ; Z=3.10$;
A.

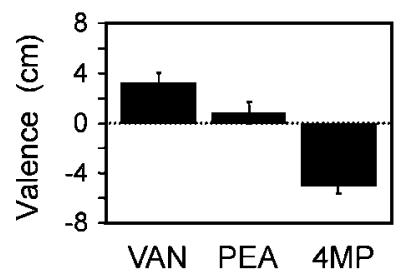

B.

c.

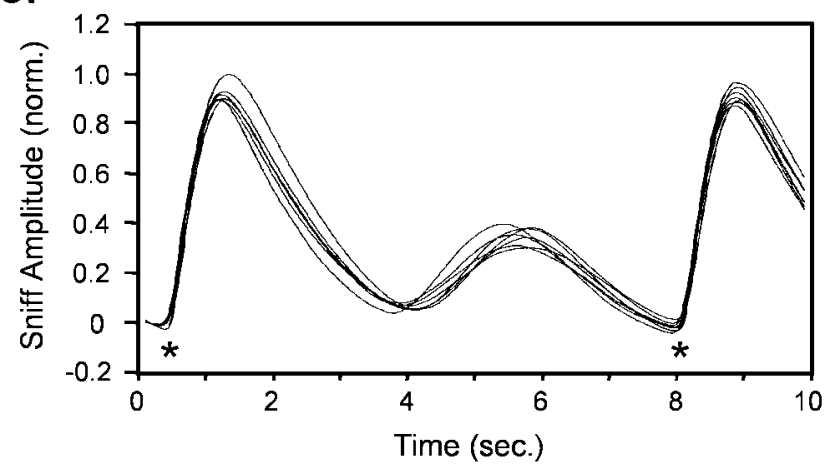

D.

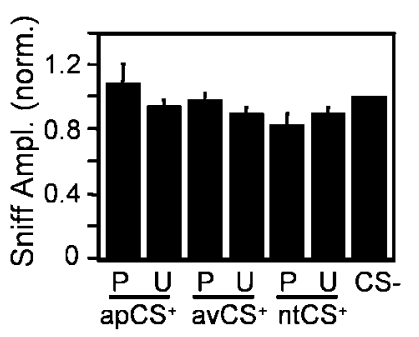

E.

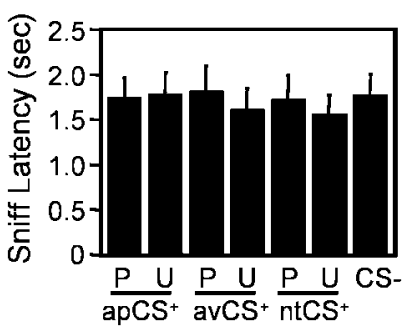

Figure 2. Behavioral results. $A$, Subjective ratings of odor valence for pleasant $(V A N)$, neutral $(P E A)$, and unpleasant $(4 M P)$ odors (mean \pm SEM; $n=15$ subjects). $B$, Mean subjective ratings of odor intensity ( \pm SEM; $n=15)$. $C$, Single-subject sniff waveforms, time-locked to trial onset, averaged over each of the seven condition types, and normalized to the $\mathrm{CS}-$ peak. An intervening respiration can be seen between two cued sniffs (marked by asterisk). D, Group mean sniff amplitudes (normalized to $\mathrm{CS}-$ ) for each condition type and collapsed across all subjects ( \pm SEM). Note that there are no error bars for the $\mathrm{CS}-$ condition because this was normalized to 1.0 for all subjects. $P$, Paired; $U$, unpaired. $E$, Group mean sniff latency (time-to-peak) for each condition ( \pm SEM).

$p<0.001$ uncorrected) and was bounded by the amygdala medially and by peri-insular cortex/claustrum laterally. On the right, activity was confined to temporal piriform cortex $(24,0,-24 ; Z=$ 2.75; $p<0.005$ uncorrected). Significant responses were also seen in bilateral amygdala (left, $-14,-10,-18 ; Z=4.41 ; p<0.05$ SVC; right, 24, $-8,-18 ; Z=3.80 ; p<0.05$ SVC), with extensive activation along the mediodorsal edge from $y=-10$ to -4 extending superiorly into ventral pallidum (Fig. 3B,D,ii). Within posterior regions of bilateral OFC, significant activations were also detected $(20,30,-20 ; Z=3.76 ; p=0.053 \mathrm{SVC}$; and $-24,34$, $-16 ; \mathrm{Z}=3.31 ; p<0.001$ uncorrected) (Fig. 3C,D, iii). Finally, in contrast to the above regions, which showed sustained responses over the course of the experiment, a conjunction of the three condition $\times$ time interactions revealed a single area of habituating response in right agranular insular cortex $(36,8,-22 ; Z=$ 4.33; $p<0.001$ uncorrected) (Table 1). 
A. i.
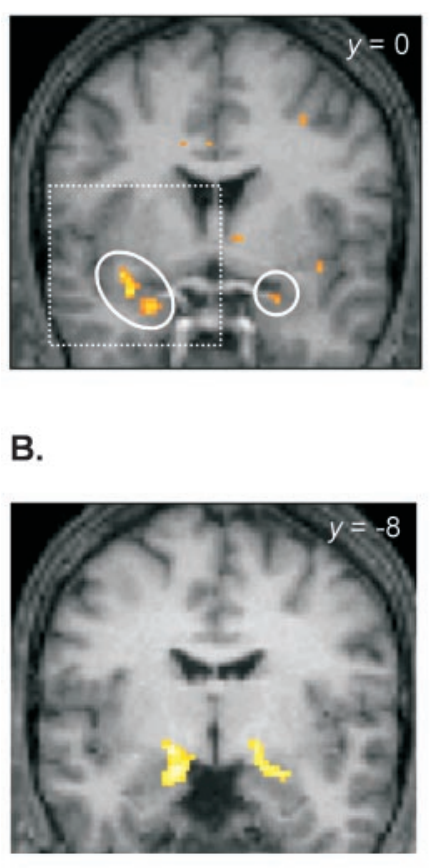

ii.

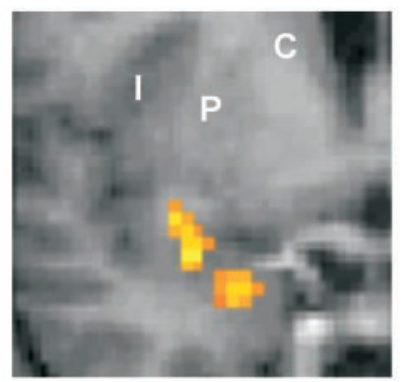

c.

iii.

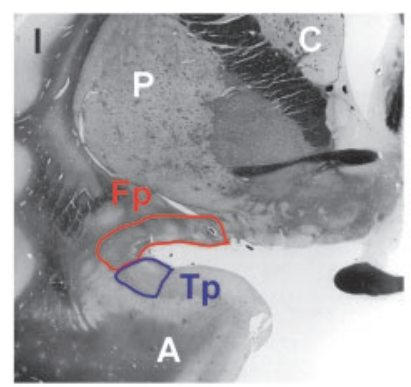

D. i. Post. piriform cortex

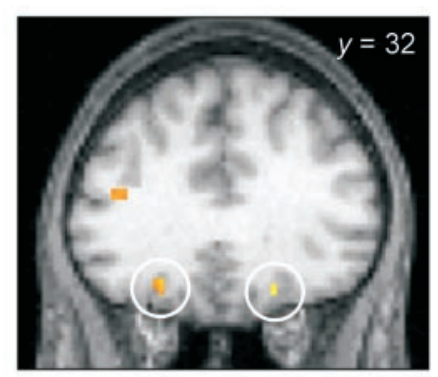

Figure 3. Valence-independent neural activations. $A$, Piriform cortex. $i$, The SPM (threshold, $p<0.005$ ) is superimposed on a subject's normalized T1weighted scan and depicts bilateral activations in posterior piriform cortex. Note in this and all subsequent figures that the left side of the brain corresponds to the left side of the figure (neurological convention). $i i, i i i$, The region bounded by the rectangle in $i$ is shown magnified in $i i$ for comparison with a high-resolution anatomical image of posterior piriform cortex (iii) [modified from Mai et al. (1997) and used with permission of Academic Press and the author]. $F p$, Frontal piriform cortex; $T p$, temporal piriform cortex; $A$, amygdala; $C$, caudate; $P$, putamen, $I$, insula. $B$, Amygdala. Neural responses in bilateral dorsomedial amygdala are shown (threshold, $p<0.001$ ). $C$, Orbitofrontal cortex. Caudal central regions of orbitofrontal cortex were bilaterally activated by all odors (threshold, $p<0.001$ ). $D$, Contrasts of parameter estimates $(\beta$, arbitrary units) were derived from each subject for each of the main effects in posterior piriform cortex (i) (at $-26,2$, -26), posterior amygdala (ii) (at -14 ,

$-10,-18)$, and centroposterior OFC

(iii) (at 20,30,-20) and collapsed across all subjects (means \pm SEM). Each of the odors, regardless of valence, elicits significant activation.

\begin{tabular}{|c|c|c|c|c|c|}
\hline \multirow[b]{2}{*}{ Brain region } & \multicolumn{3}{|c|}{ MNI coordinates $(\mathrm{mm})$} & \multirow[b]{2}{*}{ Peak $Z$} & \multirow[b]{2}{*}{$p$ value } \\
\hline & $x$ & $y$ & $z$ & & \\
\hline \multicolumn{6}{|l|}{ Main effect (conjunction) } \\
\hline Left amygdala & -14 & -10 & -18 & 4.41 & $<0.05^{*}$ \\
\hline Right amygdala & 24 & -8 & -18 & 3.80 & $<0.05^{*}$ \\
\hline Right posterior OFC & 20 & 30 & -20 & 3.76 & $0.053^{*}$ \\
\hline \multirow[t]{2}{*}{ Left posterior piriform cortex } & -32 & 4 & -20 & 3.46 & $<0.05^{*}$ \\
\hline & -26 & 2 & -26 & 3.10 & $<0.001$ \\
\hline \multirow[t]{2}{*}{ Left posterior OFC } & -24 & 34 & -16 & 3.31 & $<0.001$ \\
\hline & -22 & 24 & -20 & 3.22 & $<0.001$ \\
\hline Right agranular insula & 38 & 4 & -14 & 3.15 & $<0.001$ \\
\hline Right posterior piriform cortex & 24 & 0 & -24 & 2.75 & $<0.005$ \\
\hline \multicolumn{6}{|c|}{ Condition $\times$ time interaction (conjunction) } \\
\hline Right agranular insula & 36 & 8 & -22 & 4.33 & $<0.001$ \\
\hline
\end{tabular}

* Corrected for multiple comparisons across a small volume of interest.

\section{Activations induced by unpleasant odor}

The contrast of $\left(\right.$ avCS $+_{\mathrm{P}}-$ avCS $\left.{ }_{U}\right)$ identified olfactory-evoked responses specific to the unpleasant odor context. This revealed significant activity in the left lateral-posterior OFC $(-26,36$, $-16 ; Z=3.15 ; p<0.001$ uncorrected) (Fig. 4A) and in the right dorsal amygdala $(22,-10,-16 ; Z=3.20$; and $18,-6,-16 ; Z=$ 3.14; $p<0.001$ uncorrected). Significant activations were also observed in the left lateral hypothalamus and right insula/frontal operculum. Although piriform regions were not identified in this main effect, the contrast of condition $\times$ time interactions [namely, $\left(\right.$ avCS $\left.+_{\mathrm{P}}\right) \times$ time $-\left(\operatorname{avCS}_{\mathrm{U}}\right) \times$ time $]$ demonstrated significant habituating responses within an anterior sector of the left frontal piriform cortex $(-30,10,-28 ; Z=3.70 ; p<0.05 \mathrm{SVC})$ (Fig. 4B). This activation was $8-10 \mathrm{~mm}$ more anterior than the foci detected in the odor conjunction. Note that piriform habituation was also observed on the right side $(30,10,-28)$, but this did not reach statistical significance $(Z=2.42 ; p=0.008)$. In a direct comparison of time-modulated unpleasant $\left[\left(\mathrm{avCS}+_{\mathrm{P}}\right) \times\right.$ time $\left(\operatorname{avCS}+_{U}\right) \times$ time $]$ and pleasant $\left[\left(\operatorname{appCS}+_{P}\right) \times\right.$ time $\left(\operatorname{appCS}+_{U}\right) \times$ time] olfactory conditions, significant habituation was seen in left anterior piriform cortex and mediodorsal thalamus (Table 2), which reflected greater differential responses to unpleasant than to pleasant odor.

\section{Activations induced by pleasant odor}

Neural responses related to pleasant odor valence were evaluated by testing the main effect $\left(\operatorname{appCS}+_{P}-\operatorname{appCS}{ }_{U}\right)$. Significant activations were identified in medial anterior (frontal) piriform cortex $(24,12,-30 ; Z=3.70 ; p<0.05$ SVC) bordering the 
A. i.

Figure 4. Neural activations evoked by unpleasant odor. $A$, Orbitofrontal cortex. $i$, The main effect of unpleasant odor $\left(\mathrm{avCS}_{{ }_{\mathrm{P}}}-\mathrm{avCS}_{\mathrm{U}}\right)$ showed significant BOLD increases within left lateral posterior orbitofrontal cortex, which is superimposed on a T1weighted scan (threshold, $p<0.001$ ). $i i$, The contrasts of parameter estimates for each main effect are plotted for this region and highlight significantly greater responses to negative odor valence. $B$, Piriform cortex. $i$, Habituating neural activations in left anterior piriform cortex were revealed by contrasting the condition $\times$ time interactions $\left(\operatorname{avCS}+_{\mathrm{P}}-\mathrm{avCS}+_{\mathrm{U}}\right)$ (threshold, $p<$ 0.005). ii, Contrasts of parameter estimates in anterior piriform cortex are plotted for each odor. iii, $i v$, The brain region outlined in $i$ is magnified in $i i i$ and can be compared with a highresolution atlas image of anterior piriform cortex in iv [modified from Mai et al. (1997), and used with permission of Academic Press and the author].

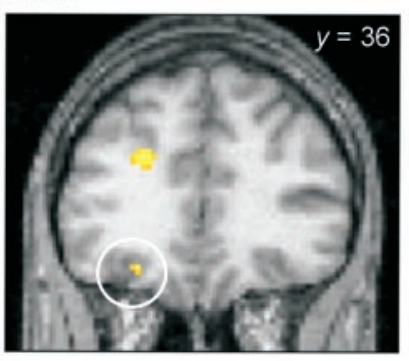

\section{A. ii.}

Posterolateral OFC $(-26,36,-16)$

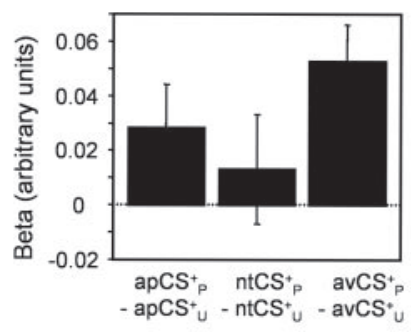

B. i.

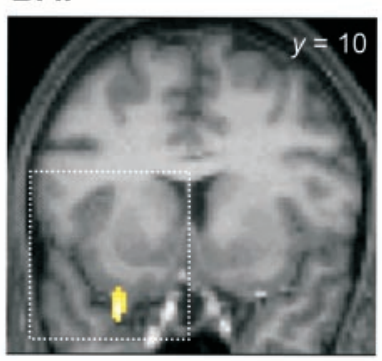

B. ii.

Ant. piriform cortex $(-30,10,-28)$

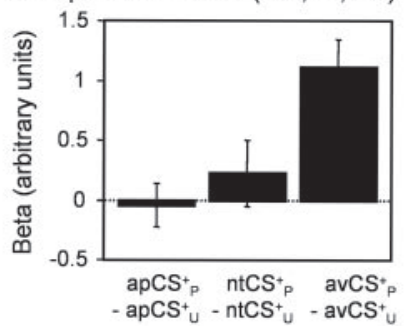

B. iii.

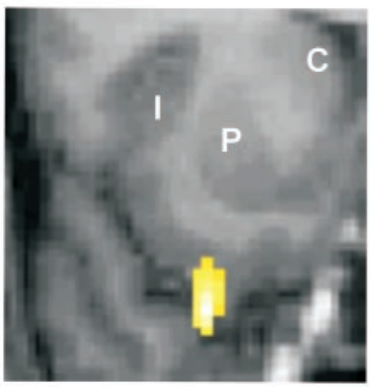

B. iv.

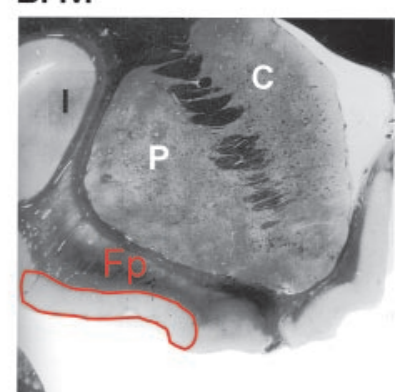

Table 2. Regions activated by unpleasant odor

MNI coordinates ( $\mathrm{mm})$

\begin{tabular}{|c|c|c|c|c|c|}
\hline Brain region & $x$ & $y$ & $z$ & Peak $Z$ & $p$ value \\
\hline \multicolumn{6}{|l|}{$\left(\operatorname{avCS}+_{P}-\operatorname{avCS}+_{U}\right)$} \\
\hline Right insula/frontal operculum & 40 & 20 & 0 & 3.43 & $<0.001$ \\
\hline Left lateral hypothalamus & -6 & -8 & -14 & 3.35 & $<0.001$ \\
\hline \multirow[t]{2}{*}{ Right amygdala } & 24 & -12 & -16 & 3.23 & $<0.001$ \\
\hline & 18 & -6 & -16 & 3.14 & $<0.001$ \\
\hline Left posterolateral OFC & -26 & 36 & -16 & 3.15 & $<0.001$ \\
\hline \multicolumn{6}{|l|}{$\left(\operatorname{avCS}+_{P}-\operatorname{avCS}_{U}\right) \times$ time } \\
\hline \multirow[t]{2}{*}{ Left anterior piriform cortex } & -30 & 10 & -28 & 3.70 & $<0.05^{*}$ \\
\hline & -30 & 6 & -30 & 3.52 & $<0.05^{*}$ \\
\hline \multicolumn{6}{|c|}{$\left(\operatorname{avCS}+_{P}-\operatorname{avCS}+_{U}\right)-\left(\operatorname{appCS}+_{P}-\operatorname{appCS}+_{U}\right) \times$ time } \\
\hline \multirow[t]{2}{*}{ Mediodorsal thalamus } & -2 & -24 & 8 & 3.62 & $<0.05^{*}$ \\
\hline & 0 & -16 & 10 & 3.59 & $<0.05^{*}$ \\
\hline \multirow[t]{2}{*}{ Left anterior piriform cortex } & -30 & 6 & -30 & 3.55 & $<0.05^{*}$ \\
\hline & -30 & 12 & -30 & 3.21 & $<0.05^{*}$ \\
\hline
\end{tabular}

* Corrected for multiple comparisons across a small volume of interest.

olfactory tubercle and extending posteriorly toward the junction of frontal and temporal cortices (Fig. 5A). A second focus of activation was found in the medial $\operatorname{OFC}(18,16,-16 ; Z=3.54$; $p<0.001$ uncorrected) (Fig. 5B). The direct comparison of $\left[\left(\operatorname{appCS}+_{P}\right)-\left(\operatorname{appCS}+_{U}\right)\right]$ to $\left[\left(\operatorname{avCS}+_{P}\right)-\left(\operatorname{avCS}+_{U}\right)\right]$ revealed significant activity within anterior piriform cortex (Table 3) driven primarily by an enhanced activation to pleasant odor. Conversely, neither of the condition $\times$ time interactions [that is, $\left(\operatorname{appCS}+_{\mathrm{P}}\right) \times$ time $-\left(\operatorname{appCS}+_{\mathrm{U}}\right) \times$ time; or $\left(\operatorname{appCS}+_{\mathrm{P}}\right) \times$ time $-\left(\operatorname{appCS}+_{U}\right) \times$ time $)-\left[\left(\operatorname{avCS}+_{P}\right) \times\right.$ time $\left(\operatorname{avCS}+_{U}\right) \times$ time $\left.)\right]$ showed significant effects. To discount the possibility that piriform cortex was simply habituating with a slower time course, the data were remodeled using a slower exponential decay-function (one-quarter session length, as opposed to one-eighth), but again no effect could be demonstrated. Thus, in contrast to the habituating profile elicited by unpleasant odor, a sustained, nonhabituating pattern of activity was observed in the presence of pleasant odor. These temporal dissociations are illustrated at the single-subject and group levels in Figure 6.

\section{Activations induced by neutral odor}

Activations specific to the neutral-valenced odor were tested by contrasting $\mathrm{ntCS}+_{\mathrm{P}}$ relative to $\mathrm{ntCS}+_{\mathrm{U}}$. Significant olfactoryevoked responses were limited to a region of left posterior piriform cortex that bordered the amygdala medially $(-20,0,-24$; $Z=3.12 ; p<0.001$ uncorrected). An adjacent piriform area $(-26,2,-24 ; Z=3.24 ; p<0.001$ uncorrected) exhibited a significant habituating response in the condition $\times$ time interaction (Table 4). Both of these activations overlapped the same regions of piriform cortex identified in the conjunction analysis described above. 
A. i.

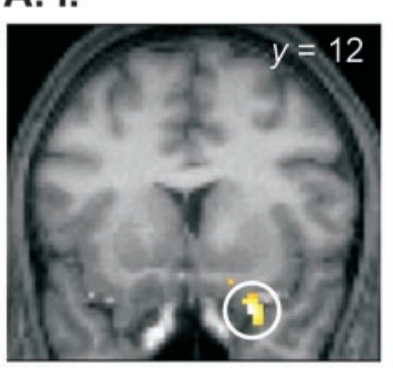

B. i.

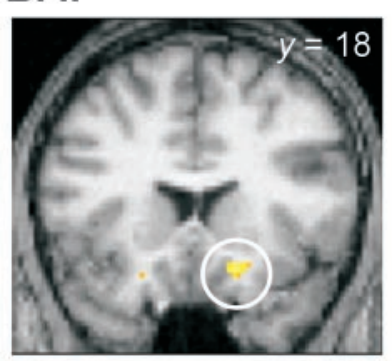

ii.

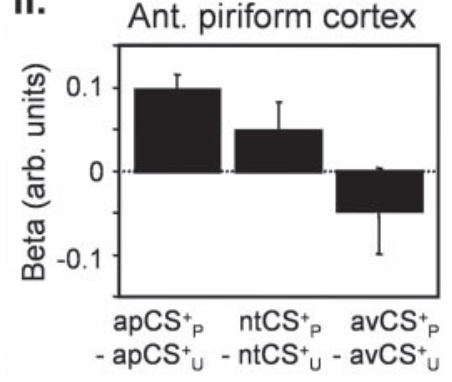

ii. Posteromedial OFC

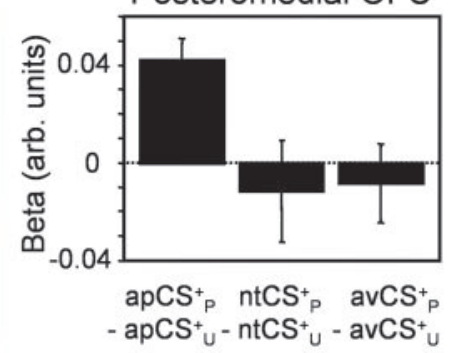

Figure 5. Neural activations evoked by pleasant odor. $A$, Piriform cortex. $i$, The main effect of pleasant odor $\left(\operatorname{appCS}+_{\mathrm{P}}-\operatorname{appCS}+_{\mathrm{U}}\right)$ showed nonhabituating responses in right anterior piriform cortex that are overlaid on a normalized T1-weighted scan (threshold, $p<0.005$ ). $i i$, Contrasts of parameter estimates in this anterior piriform region (at 24, -12 , $-30)$ are depicted for each of the main effects. $B$, Orbitofrontal cortex. $i$, Significant neural activations were also observed in right caudomedial orbitofrontal cortex (at 18, 16, -16) (threshold, $p<0.001$ ). ii, The corresponding plots of parameter estimates are charted.

\section{DISCUSSION}

The successful application of event-related techniques to olfactory fMRI, as described here, allows the implementation of more flexible experimental paradigms that are protected against changes in cognitive set, a standard confound of blocked designs (Josephs et al., 1997). In the context of olfaction, an important benefit is to reduce total stimulus exposure and limit olfactory habituation, which may have hindered detection of piriform cortex in previous neuroimaging studies. Using such approaches, we identified odor-evoked neural responses in several putative olfactory regions, including piriform cortex, amygdala, and caudal OFC. By manipulating odor valence, we observed both regional and temporal dissociations of function.

\section{Odor-evoked responses in piriform cortex}

Posterior piriform cortex was activated bilaterally by all odors, independent of valence (Fig. $3 A$ ). The significance of these effects at the group level indicates that central olfactory projections converge on an area of piriform cortex conserved across subjects. These activations persisted over a 25 min experiment, which helps validate the efficacy of event-related designs in mitigating olfactory habituation. On the basis of cognitive assumptions underlying conjunction analyses (Price and Friston, 1997), it is likely that posterior piriform cortex mediates basic odor perception and detection. Such a role complements theories derived from animal models suggesting that piriform cortex is broadly tuned to odors (Tanabe et al., 1975; Schoenbaum and Eichenbaum, 1995) and conforms to recent neuroimaging studies demonstrating similar activation patterns in response to low-level olfactory processes. In this regard, the posterior piriform activations described here are situated close (albeit slightly more lateral) to those identified in previous imaging experiments of "passive" smelling [for example, at $-21,6,-21$ (Zatorre et al., 1992); at 20, -2, -10 (Savic et al., 2000); at 24, $-1,-11$ (Sobel et al., 2000); and at $-21,0,-9$ (Poellinger et al., 2001); note that some discrepancy inevitably results in comparing MNI and non-MNI coordinate systems]. This anatomical consistency among studies supports the argument that posterior piriform cortex is critical to elementary olfactory processing.

We also observed activations within an anterior segment of piriform cortex in response to unpleasant and pleasant odors (Figs. 4B, 5A). This area corresponds to human frontal (pre)piriform cortex (Eslinger et al., 1982; Mai et al., 1997) and lies caudal to the posterior-most extent of OFC. Significant responses were seen in the main contrasts for each valence type and in direct comparisons between valence types, implying a robust effect at the group level. Thus, it appears that anterior piriform cortex is receptive to hedonic quality, especially at extremes of odor valence. The fact that piriform activations evoked by the neutral (nonvalenced) odor were comparatively restricted to posterior areas lends support to this argument. The strong reciprocal connections between anterior piriform cortex and orbitofrontal structures (Ekstrand et al., 2001) would allow ready dissemination of affective olfactory information to areas responsible for modulating behavior. Interestingly, in a recent PET study by Dade and colleagues (2002), the activation of an anatomically contiguous piriform region (at 28, 10, -21) during short-term odor recognition is in keeping with the idea of increasing specialization within more anterior segments of human olfactory cortex.

Although pleasant and unpleasant odors elicited common spatial patterns in anterior piriform cortex, their temporal patterns deviated. Anterior piriform activity was sustained in response to VAN but decreased steadily over time to 4MP (Fig. 6). This temporal dissociation may reflect physiological differences in the encoding of biologically salient olfactory information. Such an

Table 3. Regions activated by pleasant odor

\begin{tabular}{|c|c|c|c|c|c|}
\hline \multirow[b]{2}{*}{ Brain region } & \multicolumn{3}{|c|}{ MNI coordinates $(\mathrm{mm})$} & \multirow[b]{2}{*}{ Peak $Z$} & \multirow[b]{2}{*}{$p$ value } \\
\hline & $x$ & $y$ & $z$ & & \\
\hline \multicolumn{6}{|l|}{$\left(\operatorname{appCS}+_{\mathrm{P}}-\operatorname{appCS}+_{\mathrm{U}}\right)$} \\
\hline Right anterior piriform cortex & 24 & 12 & -30 & 3.91 & $<0.05^{*}$ \\
\hline Right agranular insula & 38 & 4 & -20 & 3.66 & $<0.001$ \\
\hline Right posterior orbitofrontal cortex & 18 & 16 & -16 & 3.54 & $<0.001$ \\
\hline \multicolumn{6}{|l|}{$\left(\operatorname{appCS}+_{\mathrm{P}}-\operatorname{appCS}+_{\mathrm{U}}\right)-\left(\operatorname{avCS}+_{\mathrm{P}}-\operatorname{avCS}+_{\mathrm{U}}\right)$} \\
\hline Right anterior piriform cortex & 20 & 8 & -26 & 2.95 & $<0.005$ \\
\hline
\end{tabular}

\footnotetext{
* Corrected for multiple comparisons across a small volume of interest.
} 

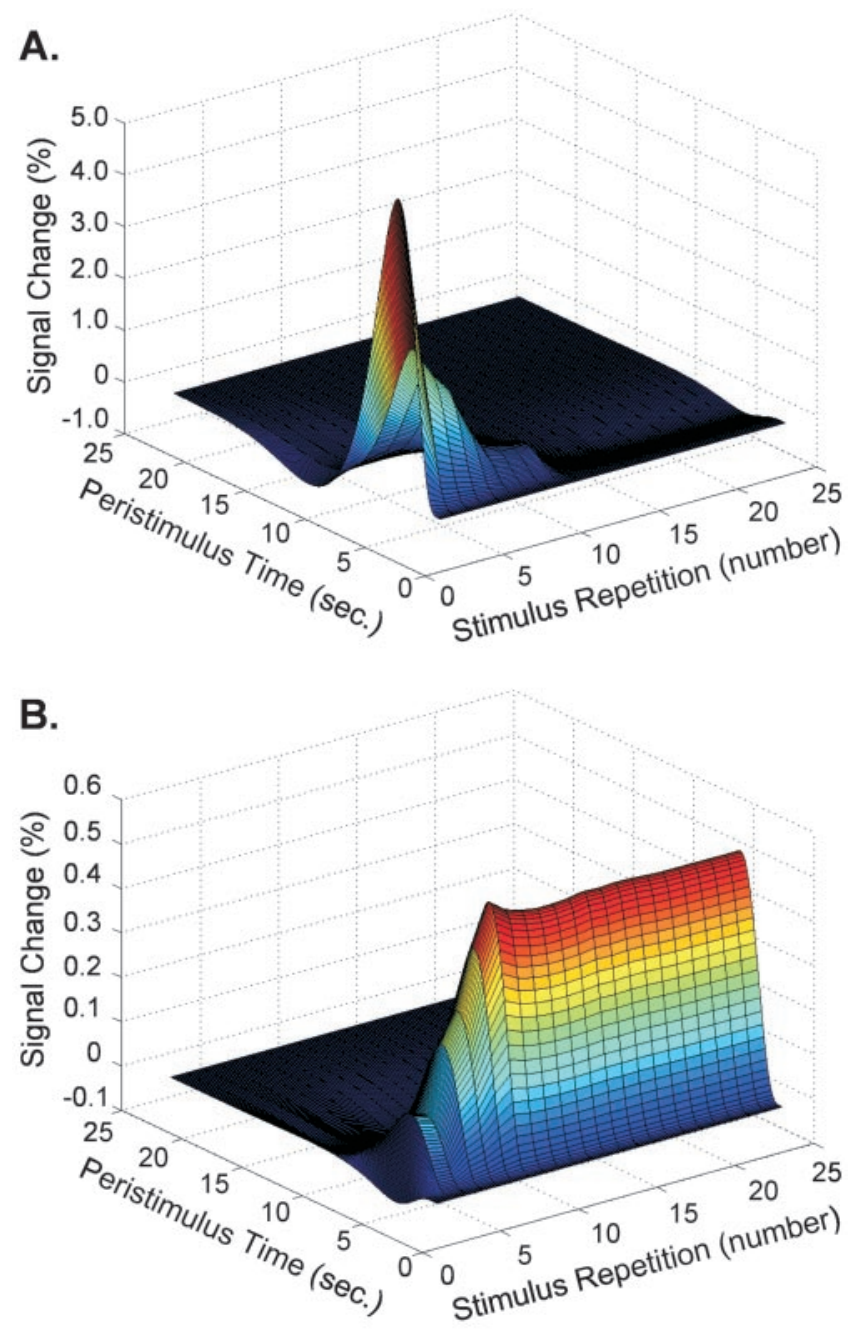

C.

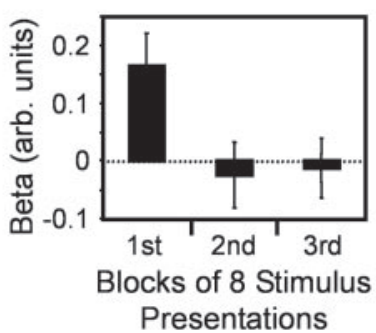

D.

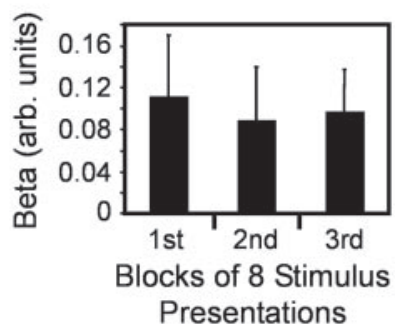

Figure 6. Temporal dynamics of olfactory processing are modulated by odor valence within anterior piriform cortex. $A, C$, Habituating neural response to unpleasant odor. $A$, The response profile in anterior piriform cortex (at $-30,10,-28)$ from a representative subject is depicted threedimensionally. With successive stimulus repetitions, the percentage

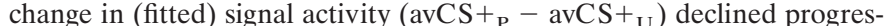
sively. $C$, The contrasts of parameter estimates for unpleasant odor were computed over three successive experimental blocks and averaged over all subjects. The BOLD activity was significantly increased during the first third of the experiment but declined markedly in the remaining twothirds. $B, D$, Sustained neural response to pleasant odor. $B$, The response profile in anterior piriform cortex (at 24, 12, -30) is shown threedimensionally from the same subject as in $A$ and reveals no decline in signal with repetitions of pleasant odor $\left(\operatorname{appCS}+_{\mathrm{P}}-\operatorname{appCS}{ }_{\mathrm{U}}\right) . D$, Contrasts of parameter estimates show persistence of neural activity over the experiment.
Table 4. Regions activated by neutral odor

\begin{tabular}{llllll} 
& \multicolumn{2}{c}{$\begin{array}{l}\text { MNI coordinates } \\
(\mathrm{mm})\end{array}$} & & \\
\cline { 2 - 4 } Brain region & $x$ & $y$ & $z$ & Peak $Z$ & $p$ value \\
\hline $\begin{array}{l}\left(\mathrm{ntCS}+{ }_{\mathrm{P}}-\mathrm{ntCS}+_{\mathrm{U}}\right) \\
\text { Left posterior piriform } \\
\quad \text { cortex }\end{array}$ & -20 & 0 & -24 & 3.12 & $<0.001$ \\
$\left(\mathrm{ntCS}{ }_{\mathrm{P}}-\mathrm{ntCS}{ }_{\mathrm{U}}\right) \times$ & & & & & \\
$\quad \begin{array}{llllll}\text { time } \\
\text { Left posterior piriform } \\
\text { cortex }\end{array}$ & -26 & 2 & -24 & 3.24 & $<0.001$ \\
\hline
\end{tabular}

idea accords with animal evidence that underscores the importance of temporal dynamics to the olfactory code (Eeckman and Freeman, 1990; Laurent, 1996) and its sensitivity to odor salience (Freeman and Schneider, 1982; Chabaud et al., 2000). Alternately, it is possible that odor valence directly influences anterior piriform habituation. In our study, whether neural habituation was exaggerated by unpleasant odor or attenuated by pleasant odor could not be determined. Nevertheless, it is worth noting that the habituating responses routinely observed in rodent olfactory bulb are blocked by reward-associated odors (Wilson and Sullivan, 1992).

These results suggest functional heterogeneity in subregions of piriform cortex. Different areas (anterior, posterior) exhibited divergent responses to the same odor (4MP), and the same area (anterior) variably responded to different odors (4MP, VAN). It is difficult to corroborate our findings with the human literature, because previous imaging studies of odor valence did not detect significant piriform activation (Zald and Pardo, 1997; Fulbright et al., 1998; Royet et al., 2001). On the other hand, anatomical and functional distinctions have been described in animal models along the rostral-caudal axis of piriform cortex (Litaudon et al., 1997; Haberly, 1998; Chabaud et al., 2000). Moreover, activity in olfactory bulb and cortex can be modulated by biologically meaningful odors (Chabaud et al., 2000) and behaviorally conditioned odors (Pager, 1974; Freeman and Schneider, 1982; Wilson and Sullivan, 1992). Our findings extend the validity of these concepts to human olfactory processing.

\section{Odor-evoked responses in amygdala}

The odor conjunction revealed extensive, and sustained, activation in the amygdala along its dorsomedial sector (Fig. 3B). This spatial pattern accords with the known distribution of olfactory efferents in animal models of amygdala. Projections from olfactory bulb and piriform cortex terminate in periamygdaloid cortex, anterior and posterior cortical nuclei, and medial nucleus (Carmichael et al., 1994), which overlap the activations described here. It is interesting to note that, in contrast to other sensory modalities, olfaction has no direct inputs into the lateral nuclei. Although it can be argued that the observed activations spare lateral amygdala, we would caution against too strong an inference, given the limits of spatial resolution imposed by our technique.

Neurophysiological recordings in animals (Cain and Bindra, 1972; Tanabe et al., 1975) and humans (Hughes and Andy, 1979; Hudry et al., 2001) suggest that the amygdala is broadly tuned to various odors. From an evolutionary perspective, the physical expansion of primate amygdala paralleled increases in paleocortex, mostly comprising piriform cortex, and consequently much of 
the amygdala was committed to olfactory processing (Barton and Aggleton, 2000). Despite a relative decline in human odor sensibility, our findings suggest that much of this circuitry is still functional. Note that although amygdala responded to all odors, independent of hedonic content, neural activity was more pronounced in the presence of unpleasant odor. This was evident in the conjunction analysis, where markers of neural activity ( $\beta$ values) showed an increasing trend with increasing odor aversiveness (Fig. 3D,ii) and in the main effect of unpleasant odor. Thus, odor-evoked activity in the amygdala is not restricted to aversiveodor processing but exhibits a tendency in that direction, which complements notions that the amygdala may show preferential involvement in negative emotional processing of both olfactory (Hughes and Andy, 1979; Zald and Pardo, 1997) and nonolfactory (Morris et al., 1996; Zald et al., 1998) stimuli.

\section{Odor-evoked responses in orbitofrontal cortex}

Significant responses were revealed by the odor conjunction in caudal OFC (Fig. 3C). These activations correspond to the socalled "central-posterior orbitofrontal cortex" (CPOF) identified by Yarita et al. (1980), who considered it a broadly tuned area of primate olfactory association cortex. According to Carmichael et al. (1994), the CPOF is roughly homologous to orbital areas $13 \mathrm{~m}$, $13 \mathrm{a}$, and Iam and represents the primary prefrontal locus of olfactory input. By hosting a convergence of primary sensory, visceral, and limbic information, olfactory OFC may enable stimulus-reward associations and the organization of odor-guided behavior (Carmichael et al., 1994). Single-unit recordings in animals show that OFC responds to complex aspects of olfactory processing (Schoenbaum and Eichenbaum, 1995; Critchley and Rolls, 1996). In humans, olfactory discrimination is impaired in patients with OFC lesions (Potter and Butters, 1980; Zatorre and Jones-Gotman, 1991), and the OFC, particularly caudal regions, is detected consistently in olfactory neuroimaging studies (Zald and Pardo, 2000). The data presented here offer further evidence that posterior OFC is a critical component of human olfaction.

The regional patterns of activation within caudal OFC diverged according to valence. Unpleasant odor evoked activity in (left) lateral-central OFC (Fig. 4A); pleasant odor evoked activity in (right) medial OFC (Fig. 5B). Similar to piriform cortex, this suggests a segregation of odor valence processing within caudal OFC. There is limited evidence in primates that medial and lateral portions of OFC are functionally distinct (Carmichael and Price, 1996), and human imaging data illustrate such dissociations. Medial OFC activity correlates with numerous measures of pleasantness such as monetary reinforcers (O'Doherty et al., 2001), tastes (Small et al., 2001), and facial attractiveness (O'Doherty et al., 2003), whereas lateral OFC responses are more aligned with negative or punishing aspects of these stimuli. Our findings indicate that this concept is applicable to the olfactory domain.

Although formal side $\times$ condition interactions were beyond the scope of this report, it is worth reemphasizing that unpleasant odor was associated with left-sided OFC activation. Similarly, in their PET study of aversive olfactory stimulation, Zald and Pardo (1997) observed a comparable pattern of activity in this region, whereas Royet et al. (2001) reported left OFC activation during odor judgments of hedonic quality. Given these findings, it is plausible that left OFC is preferentially activated when hedonic features dominate olfactory perception.

Nevertheless, it is important to note that nonhedonic differences between the three odorants, such as edibility, familiarity, or nameability, may also contribute to the differential activation patterns described not only in OFC but also in piriform cortex and amygdala. For example, the VAN-evoked neural activity could have been equally a product of odor "vanilla-ness" as odor pleasantness, thereby activating OFC regions independent of hedonic valence. As such, our conclusions regarding functional dissociations along the hedonic dimension are tentative.

Olfactory references to Proust's (1913) tea-soaked madeleines are often summoned as prima facie proof for an emotional primacy of odors. It is also claimed that the structural overlap of olfactory and limbic structures necessarily dictates an intimate functional relationship. However, although odor perception appears to be dominated by hedonic qualities (Schiffman, 1974), there is little anatomical evidence connecting human olfaction to emotional states. Our study lends biological credence to the link between olfaction and emotion-based processes in so far as neural representations of pleasant and unpleasant odor are spatially and temporally separable within key olfactory structures and within areas implicated in emotional processing. The fact that affective properties of odors are encoded within primary olfactory cortex affirms the idea that hedonic value is an important determinant of odor identity.

\section{REFERENCES}

Barton RA, Aggleton JP (2000) Primate evolution and the amygdala. In: The amygdala: a functional analysis (Aggleton JP, ed), pp 479-508. New York: Oxford UP.

Cain DP, Bindra D (1972) Responses of amygdala single units to odors in the rat. Exp Neurol 35:98-110.

Carmichael ST, Price JL (1996) Connectional networks within the orbital and medial prefrontal cortex of macaque monkeys. J Comp Neurol 371:179-207.

Carmichael ST, Clugnet MC, Price JL (1994) Central olfactory connections in the macaque monkey. J Comp Neurol 346:403-434.

Chabaud P, Ravel N, Wilson DA, Mouly AM, Vigouroux M, Farget V, Gervais R (2000) Exposure to behaviourally relevant odour reveals differential characteristics in rat central olfactory pathways as studied through oscillatory activities. Chem Senses 25:561-573.

Constable RT, Spencer DD (1999) Composite image formation in z-shimmed functional MR imaging. Magn Reson Med 42:110-117.

Critchley HD, Rolls ET (1996) Olfactory neuronal responses in the primate orbitofrontal cortex: analysis in an olfactory discrimination task. J Neurophysiol 75:1659-1672.

Dade LA, Zatorre RJ, Jones-Gotman M (2002) Olfactory learning: convergent findings from lesion and brain imaging studies in humans. Brain 125:86-101.

Deichmann R, Turner R (2002) Improvement of local BOLD sensitivities in the presence of susceptibility gradients by using tilted slices. Proc Int Soc Magn Reson Med 10:1414.

Doty RL, Brugger WE, Jurs PC, Orndorff MA, Snyder PJ, Lowry LD (1978) Intranasal trigeminal stimulation from odorous volatiles: psychometric responses from anosmic and normal humans. Physiol Behav 20:175-185.

Duvernoy HM (1999) The human brain: surface, blood supply, and three-dimensional sectional anatomy. Vienna: Springer.

Eeckman FH, Freeman WJ (1990) Correlations between unit firing and EEG in the rat olfactory system. Brain Res 528:238-244.

Ekman P, Friesen WV (1976) Pictures of facial affect. Palo Alto, CA: Consulting Psychologists Press.

Ekstrand JJ, Domroese ME, Johnson DM, Feig SL, Knodel SM, Behan M, Haberly LB (2001) A new subdivision of anterior piriform cortex and associated deep nucleus with novel features of interest for olfaction and epilepsy. J Comp Neurol 434:289-307.

Eskenazi B, Cain WS, Novelly RA, Friend KB (1983) Olfactory functioning in temporal lobectomy patients. Neuropsychologia 21:365-374.

Eslinger PJ, Damasio AR, Van Hoesen GW (1982) Olfactory dysfunction in man: anatomical and behavioral aspects. Brain Cogn 1:259-285.

Freeman WJ, Schneider W (1982) Changes in spatial patterns of rabbit olfactory EEG with conditioning to odors. Psychophysiology 19:44-56.

Friston KJ, Ashburner J, Frith CD, Poline J-B, Heather JD, Frackowiak RSJ (1995a) Spatial registration and normalization of images. Hum Brain Mapp 2:165-189.

Friston KJ, Holmes AP, Worsley KJ, Poline J-P, Frith CD, Frackowiak RSJ (1995b) Statistical parametric maps in functional imaging: a general linear approach. Hum Brain Mapp 2:189-210.

Friston KJ, Fletcher P, Josephs O, Holmes A, Rugg MD, Turner R 
(1998) Event-related fMRI: characterizing differential responses. NeuroImage 7:30-40.

Fulbright RK, Skudlarski P, Lacadie CM, Warrenburg S, Bowers AA, Gore JC, Wexler BE (1998) Functional MR imaging of regional brain responses to pleasant and unpleasant odors. AJNR Am J Neuroradiol 19:1721-1726.

Glaser D, Penny W, Henson R, Friston KJ (2002) Coping with the variance structure of neuroimaging data. Abstr Cogit Neurosci Soc 9:141.

Gottfried JA, O'Doherty J, Dolan RJ (2002) Appetitive and aversive olfactory learning in humans studied using event-related functional magnetic resonance imaging. J Neurosci 22:10829-10837.

Haberly LB (1998) Olfactory cortex. In: The synaptic organization of the brain (Shepherd GM, ed), pp 377-416. New York: Oxford UP.

Hudry J, Ryvlin P, Royet JP, Mauguiere F (2001) Odorants elicit evoked potentials in the human amygdala. Cereb Cortex 11:619-627.

Hughes JR, Andy OJ (1979) The human amygdala. I. Electrophysiological responses to odorants. Electroencephalogr Clin Neurophysiol 46:428-443.

Josephs O, Turner R, Friston K (1997) Event-related fMRI. Hum Brain Mapp 5:243-248.

Josephs O, Deichmann R, Turner R (2000) Trajectory measurement and generalized reconstruction in rectilinear EPI. Proc Int Soc Magn Reson Med 8:1517.

Kobal G, Hummel C (1991) Olfactory evoked potentials in humans. In: Smell and taste in health and disease (Getchell TV, Doty RL, Bartoshuk LM, Snow JB, eds), pp 255-275. New York: Raven.

Laing DG (1986) Identification of single dissimilar odors is achieved by humans with a single sniff. Physiol Behav 37:163-170.

Laurent G (1996) Dynamical representation of odors by oscillating and evolving neural assemblies. Trends Neurosci 19:489-496.

Litaudon P, Datiche F, Cattarelli M (1997) Optical recording of the rat piriform cortex activity. Prog Neurobiol 52:485-510.

Lorig TS, Elmes DG, Zald DH, Pardo JV (1999) A computer-controlled olfactometer for fMRI and electrophysiological studies of olfaction. Behav Res Methods Instrum Comput 31:370-375.

Mai JK, Assheuer J, Paxinos G (1997) Atlas of the human brain. San Diego: Academic.

Morris JS, Frith CD, Perrett DI, Rowland D, Young AW, Calder AJ, Dolan RJ (1996) A differential neural response in the human amygdala to fearful and happy facial expressions. Nature 383:812-815.

O'Doherty J, Kringelbach ML, Rolls ET, Hornak J, Andrews C (2001) Abstract reward and punishment representations in the human orbitofrontal cortex. Nat Neurosci 4:95-102.

O'Doherty J, Winston JS, Critchley H, Perrett D, Burt DM, Dolan RJ (2003) Beauty in a smile: the role of medial orbitofrontal cortex in facial attractiveness. Neuropsychologia 41:147-155.

Ojemann JG, Akbudak E, Snyder AZ, McKinstry RC, Raichle ME, Conturo TE (1997) Anatomic localization and quantitative analysis of gradient refocused echo-planar fMRI susceptibility artifacts. NeuroImage 6:156-167.

Pager J (1974) A selective modulation of the olfactory bulb electrical activity in relation to the learning of palatability in hungry and satiated rats. Physiol Behav 12:189-195.

Poellinger A, Thomas R, Lio P, Lee A, Makris N, Rosen BR, Kwong KK
(2001) Activation and habituation in olfaction — an fMRI study. NeuroImage 13:547-560.

Potter H, Butters N (1980) An assessment of olfactory deficits in patients with damage to prefrontal cortex. Neuropsychologia 18:621-628.

Price CJ, Friston KJ (1997) Cognitive conjunction: a new approach to brain activation experiments. NeuroImage 5:261-270.

Proust M (1913) Swann's way (Moncrieff CKS, translator). New York: Modern Library, 1956.

Rorden C, Brett M (2000) Stereotaxic display of brain lesions. Behav Neurol 12:191-200.

Royet JP, Hudry J, Zald DH, Godinot D, Gregoire MC, Lavenne F, Costes N, Holley A (2001) Functional neuroanatomy of different olfactory judgments. NeuroImage 13:506-519.

Savic I, Gulyas B, Larsson M, Roland P (2000) Olfactory functions are mediated by parallel and hierarchical processing. Neuron 26:735-745.

Schiffman SS (1974) Physicochemical correlates of olfactory quality. Science 185:112-117.

Schoenbaum G, Eichenbaum H (1995) Information coding in the rodent prefrontal cortex. I. Single-neuron activity in orbitofrontal cortex compared with that in pyriform cortex. J Neurophysiol 74:733-750.

Small DM, Zatorre RJ, Dagher A, Evans AC, Jones-Gotman M (2001) Changes in brain activity related to eating chocolate: from pleasure to aversion. Brain 124:1720-1733.

Sobel N, Prabhakaran V, Desmond JE, Glover GH, Goode RL, Sullivan EV, Gabrieli JD (1998) Sniffing and smelling: separate subsystems in the human olfactory cortex. Nature 392:282-286.

Sobel N, Prabhakaran V, Zhao Z, Desmond JE, Glover GH, Sullivan EV, Gabrieli JD (2000) Time course of odorant-induced activation in the human primary olfactory cortex. J Neurophysiol 83:537-551.

Tanabe T, Iino M, Takagi SF (1975) Discrimination of odors in olfactory bulb, pyriform-amygdaloid areas, and orbitofrontal cortex of the monkey. J Neurophysiol 38:1284-1296.

Wilson DA (1998) Habituation of odor responses in the rat anterior piriform cortex. J Neurophysiol 79:1425-1440.

Wilson DA, Sullivan RM (1992) Blockade of mitral/tufted cell habituation to odors by association with reward: a preliminary note. Brain Res 594:143-145.

Worsley KJ, Marrett S, Neelin P, Vandal AC, Friston KJ, Evans AC (1996) A unified statistical approach for determining significant voxels in images of cerebral activation. Hum Brain Mapp 4:58-73.

Yarita H, Iino M, Tanabe T, Kogure S, Takagi SF (1980) A transthalamic olfactory pathway to orbitofrontal cortex in the monkey. J Neurophysiol 43:69-85.

Zald DH, Pardo JV (1997) Emotion, olfaction, and the human amygdala: amygdala activation during aversive olfactory stimulation. Proc Natl Acad Sci USA 94:4119-4124.

Zald DH, Pardo JV (2000) Functional neuroimaging of the olfactory system in humans. Int J Psychophysiol 36:165-181.

Zald DH, Lee JT, Fluegel KW, Pardo JV (1998) Aversive gustatory stimulation activates limbic circuits in humans. Brain 121:1143-1154.

Zatorre RJ, Jones-Gotman M (1991) Human olfactory discrimination after unilateral frontal or temporal lobectomy. Brain 114:71-84.

Zatorre RJ, Jones-Gotman M, Evans AC, Meyer E (1992) Functional localization and lateralization of human olfactory cortex. Nature 360: $339-340$. 